\title{
The Role of Design Thinking and Physical Prototyping in Social Software Engineering
}

\author{
Peter Newman*, Maria Angela Ferrario ${ }^{\dagger}$, Will Simm*, Stephen Forshaw ${ }^{\ddagger}$, Adrian Friday*, and Jon Whittle* \\ ${ }^{*}$ School of Computing and Communications, ${ }^{\dagger}$ Lancaster University Management School, ${ }^{\ddagger}$ LICA \\ Lancaster University, UK \\ \{p.newman,m.a.ferrario,w.a.simm,s.forshaw,a.friday,j.n.whittle\}@lancaster.ac.uk
}

\begin{abstract}
Social Software Engineering (Social SE), that is SE aiming to promote positive social change, is a rapidly emerging area. Here, software and digital artefacts are seen as tools for social change, rather than end products or 'solutions'. Moreover, Social SE requires a sustained buy-in from a range of stakeholders and end-users working in partnership with multidisciplinary software development teams often at a distance. This context poses new challenges to software engineering: it requires both an agile approach for handling uncertainties in the software development process, and the application of participatory, creative design processes to bridge the knowledge asymmetries and the geographical distances in the partnership. This paper argues for the role of design thinking in Social SE and highlights its implications for software engineering in general. It does so by reporting on the contributions that design thinkingand in particular physical design-has brought to (1) the problem space definition, (2) user requirements capture and (3) system feature design of a renewable energy forecasting system developed in partnership with a remote Scottish Island community.
\end{abstract}

\section{INTRODUCTION}

In prior work [1], we defined Social Software Engineering (Social SE) as the development and maintenance of software with the aim of promoting a positive change in society. In Social SE, software and digital artefacts are not just seen as an end-goal, but also as vehicles for exploring mechanisms to facilitate social change. An agile and participatory approach to software development plays a key role in the success of Social SE, as it helps with managing the uncertainties and explore the different perspectives of ever changing complex social problems [2]

Speedplay [1], a Social SE framework that integrates agile, action research and participatory design, emerged from community-based software innovation research and has been successfully applied to a number of Social SE projects addressing complex societal issues [3], [4]. Furthermore, design thinking [5] has been extensively used in complex problems exploration because it facilitates the generation of the different solutions to the same problem. For this, design thinking have been integrated within the existing Speedplay agile and participatory framework, in order to jointly explore the problem domain and creatively facilitate requirements capture.

To illustrate this approach we use a short but intense ninemonth sustainability project called OnSupply as a case study. OnSupply involved working in partnership with a community to explore the potential for synchronising daily domestic energy use to the availability of an energy supply which includes a substantial time-varying renewable energy component. Energy use in the home, and indeed it's production, distribution and consumption, is normally invisible to householders: synchronising the energy use implicit in domestic practice with sustainable supply thus represents a complex socio-technical problem domain that has a number of possibly conflicting constraints and no single or immediately obvious technical solution [6]. Our approach resulted in a relatively substantial number (12) of physical and digital technology prototypes. Each prototype enabled exploration of different aspects of the problem space, helping us build a more complete and in depth picture of the role of energy in the island context in question, and to elicit new understandings and requirements.

Like Burnett and Myers, we argue that it is not good enough to simply present the community with a technological solution to a complex problem [7]; instead, the problem space needs to be jointly explored with the community as an ongoing requirements elicitation process. Here both researchers and community participants were equally domain 'non-experts', gaining domain knowledge together in a process that develops mutual trust through skill sharing and prototype development.

In this paper we firstly present the design thinking model adopted in the SE process. We then introduce some of the physical and digital artefacts developed, and finally focus on the refinement and adoption of the most developed of our prototypes: the Tiree Energy Pulse application as an example of the technology prototypes co-developed with the community.

Our contribution to SE is embedding design thinking in an agile and participatory framework [1] that is applied to a poorly-understood complex social problem. We argue that by doing so this approach provides a powerful mechanism for the generation and development of software solutions to underspecified and open ended problem domains. In the context of complex problems, the journey to elicit requirements and user stories presents a number of challenges to the participating user; namely, writing user stories for a problem space that has yet to manifest itself [8], and their unfamiliarity with the design and requirements elicitation process [9].

We name the design thinking component of this process DivingBoard. Part of this process involves applying a design attitude (i.e. embracing discontinuity and openendedness [10]) to a complex problem exploration in an iterative build cycle. DivingBoard uses creative workshops to elicit ideas and 
themes from the participants, and then builds physical and digital prototypes that represent and communicate these ideas.

We illustrate how DivingBoard has been applied to OnSupply and describe our experience of iteratively developing several ideas with the community. As part of our exploration, we use physical artefacts, low-fidelity digital prototypes, and scenario-based games to immerse the participants in the problem context by making the intangible, tangible-that is, enabling the participants to physically engage with the problem first-hand. During this case study, a number of ideas were elicited from the community, which were developed into physical representations and software prototypes. The Tiree Energy Pulse, a renewable energy forecasting web application, is one of these prototypes and will be introduced to specifically illustrate its underpinning community co-development process.

\section{BACKGROUND}

\section{A. Designing with Rather than for the User}

User participation has long been recognised as a key element to the success of designing any software system [11] and is a key tenet of the agile approach to software development [12]. In this paper we will pay particular attention to Participatory Design (PD) [13] and its intersection with design thinking for software development (we cover the intersection between PD and agile [14]-[16] in our previous work [1]).

The origins of PD can be traced back to the 1970s in Scandinavia as a way to empower workers and foster democracy in the workplace [17]. While User-centred Design (UCD) might use the end user as the object of attention in the design of a system, PD takes this further and involves the end user in the actual design process. This difference becomes apparent in the description of PD given by Sanders and Stappers:

"[It is] a democratic approach that views users as partners stressing the importance of the social dimension of work with technology" [18].

Therefore, in $\mathrm{PD}$, emphasis is placed on the partnership between end-user and designer, and implies that both parties will work together in the design of the end product.

The involvement of the end-user in the development of new systems has also been of interest in the software community since the early 1980s [19], and forms an important part of the software development cycle [20]. More than simply being part of the requirements process, there are also initiatives that are interested in integrating user participation with existing software engineering methods, such as agile [14].

\section{B. Designing for 'Wicked' Problems}

It could be said that the term design eludes definition, as it is applied to innumerable domains (e.g. graphics, process, system etc.) spanning different parts of their respective processes. For simplicity, we will narrow down the scope of this argument and use the following working definition for design thinking as "an approach that uses creative designers' sensibility and methods to understand the problems that people and society are dealing with". In the past, there have been attempts to map design thinking onto a linear model, separating the design process into two distinct phases: problem definition and problem solution [21]. Whilst this formalised view of the design process may work for the solution of logical, deterministic problems, there is a class of problem that requires more intuition than logical analysis—wicked problems [2].

Whilst most problems encountered day-to-day may be categorised as tame, important, domain spanning complex problems, such as promoting sustainability, are frequently categorised as wicked [22]. The term wicked problem was first coined by Rittel and Webber, who outlined the 10 properties of wicked problem [6]; or, more concisely:

\section{"They are only loosely formulated. There is no stop- ping rule. Wicked problems persist, and are subject to redefinition and resolution in different ways over time [.. ]" [22].}

This description hints at the non-deterministic nature of wicked problems, meaning that designing to address them requires a certain level of designer intuition - that being said, it is important for the designer to ground any design in the context in which it is deployed. Sustainability is one such wicked problem, and synchronising with renewable energy supply is one component of a sustainable future. Here we embed design thinking in the OnSupply project through our DivingBoard component of Speedplay.

\section{DivingBoARd: ExPloring THE PROBlem}

The development of software is often criticised as following the mantra of "build it and they will come" [7], where the solution has already been envisaged by the software practitioner. Instead, users should be considered as experts of their own experience, and be involved in the exploration of the problem and solution space, as they will be on the ground, potentially using this system in their daily lives. The Speedplay framework is characterized by a participatory and agile "plan, act and reflect" process across four distinct and overlapping development steps (prepare, design, build, and sustain) [1].

The DivingBoard creative approach is particularly useful during the prepare and design steps of the Speedplay process, which is aimed at facilitating skill sharing, team building, creative problem exploration, and the rapid prototyping of ideas and possible solutions. DivingBoard uses user participation to explore the problem space with a group of non-expert end users even before requirements are elicited. Once the problem space has been explored by the participants, DivingBoard then facilitates the elicitation of requirements through a number of artefact-driven workshops.

\section{A. DivingBoard Overview}

DivingBoard follows an iterative pattern akin to the usercentred design cycle [23] (see Figure 1). Starting from an initial problem description, the DivingBoard process facilitates the exploration of the problem domain with the participants through a number of creative workshops, interviews, and meetings with stakeholders and other members of the community. Once a number of high level themes and ideas are elicited from the community, the process then involves the creation of a 


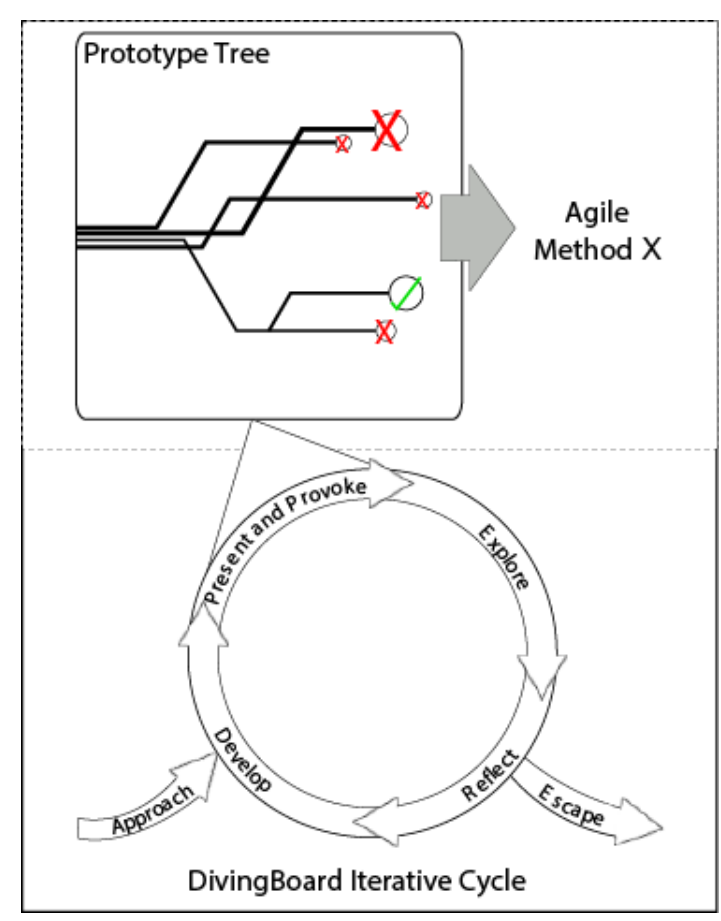

Fig. 1: Overview of the DivingBoard process.

number of prototypes that represent the aforementioned ideas. These ideas are then presented back to the participants for critical reflection and refinement; it is during this refinement that branches of the prototype tree will be either culled or further developed. The steps of DivingBoard are briefly described below:

- Approach. During this first meeting, it is important for both researchers and stakeholders to share their own history [24]; stakeholders can provide contextual information about the community, and the researchers can give insight into their methodology and experience.

- Develop. By building on artefacts and ideas presented at previous workshops and from the community feedback, low-fidelity artefacts can be developed. In the case of the first workshop, the presented artefacts are designed to give the participants a flavour of what technology are available or could be developed.

- Present and Provoke. During this step, the facilitators are able to present the latest iteration of the physical prototypes, which are intended to encourage further ideation from the participants through discussion and feedback about the artefacts.

- Explore. The Explore step facilitates the exploration of the problem space with the community participants, and also gives them a chance to share further contextual information about the community. The physical artefacts should be used in this stage as boundary objects-that is, used to help focus the problem space and provide a technological scope. In addition, other activities should be undertaken to help participants better visualise the problem space (e.g. scenario-based games and tasks).

- Reflect. Reflecting upon the feedback and other input given by participants is crucial to the develop phase, as it allows the researchers to improve upon or develop new artefacts. Here both researchers and participants reflect on mutual feedback and on subjects covered by the previous workshop.

- Escape. Once the problem has been explored and a list of requirements has been elicited, the development of prototypes attempting to address the problem space can begin (i.e. reach escape velocity). The DivingBoard process can be reentered generatively to further develop these prototypes, or explore other avenues of the problem or solution space.

To reiterate the sentiment of Kamil Michlewski [10], it is important that during this process the facilitators embrace discontinuity and openendedness - that is, not to obsess about keeping the prototypes as linear progressions of an idea, and try to resist ushering the participants towards a solution too early. While there is no formal definition of what too early might be, a reasonable heuristic from our experience is that the time to start synthesising a solution will be indicated by a reasonable level of joint understanding of the problem space, a number of artefacts having been generated, and their mapping into no more than 2-3 branches (design themes). Some prototypes may be short-lived and others will evolve through development, and participants should be prepared to discard or change an idea, and re-enter DivingBoard, if the prototype proves unsuccessful.

\section{B. Physical Artefacts}

Most agile methods already feature "physical artefacts" as part of the development process [25]; however, in this context, the physical artefacts tend to be collaboration boards and user story cards. Whilst these artefacts have an important role in facilitating collaboration between the design team, they aren't intended to inspire ideation from the end-user participants; rather, they are often generated as part of their output.

Although DivingBoard does not explicitly require the use of physical artefacts in order to elicit the generation of prototypes, we argue that they can play an important role in helping the participants understand the problem domain. In this paper, the authors define physical artefacts as high level ideas and themes represented as low-fidelity physical manifestations. As such, they are different from prototypes as they may not represent a solution, but instead present an object that may inspire discussion and visualise earlier discussed topics.

Our use of physical artefacts and game-based scenarios is intended to make the intangible, tangible, in this case appreciating the mix of energy generation and its availability, allowing the participants to physically engage with the problem context; this brings an element of learning through experience and concrete example, which are important parts of the learning cycle [26]. The artefacts in DivingBoard are also intended to provide focal points to the design process, enabling the design 
team to showcase their progress to the community, and gives further opportunity for the community to provide feedback. With the exception of the first workshop, each workshop relies on the output of the session that preceded it, and each physical artefact brought by the facilitators should be built upon the ideas or artefacts presented previously, continuing the ongoing creative dialogue.

\section{OnSupply: A Case Study}

Tiree is a remote Scottish island located in the outermost of the Inner Hebrides. Literally on the edge of the electricity grid of the UK mainland, the island is subject to frequent power-cuts and occasional shortages of supply during periods of extreme weather. In a move towards becoming more selfsufficient, the Tiree community erected a $910 \mathrm{~kW}$ E-44 Enercon wind turbine ${ }^{1}$, which is capable of delivering over $50 \%$ of the island's energy demand during periods of high wind.

As strong, prevailing winds are commonplace, the islanders have an innate connection with their environment, and frequently use their experience to predict ferry and plane cancellations due to weather conditions; often planning their food shopping and travel according to the weather. This setting affords the Tiree community an almost innate expertise and awareness of their consumption of local resources and a hightened sense of the availability of food, water, and electricity.

Following the early stages of Speedplay, we applied DivingBoard as a creative means to explore with members of the Tiree community possible digital mechanisms that can help communities adapt to an energy landscape where resources are not continuously available. We ran a series of design workshops at approximately 3 week intervals.

Due to the remoteness and inaccessibility of Tiree, and for reasons of time and also financial necessity, the authors would spend concentrated times on the island in the week surrounding each of the workshops-these engagements were significant and intensive engagements, adding up to around 240 person-days on the island over the 9 month project.

\section{A. Approaching the Community}

The first few months of the OnSupply project served two purposes: to learn about the context of the community, and to provide an opportunity for the community to meet us and learn about our history and the way we work.

During the first stakeholder meeting, we brought a portfolio of technology solutions created for other projects to illustrate our competencies and engage in a fact-finding discussion about the the partnership skillset and experience.

To capture the energy usage behaviours of the community and how they adapted to the intermittent power outages, we conducted in depth interviews with several members of the island community. From these interviews came a strong sense of community resilience but also the fragility of the communication infrastructure of the island.

\footnotetext{
${ }^{1}$ Turbine specification-http://www.enercon.de/en-en/60.htm, accessed on April 7, 2015.
}

This almost ethnographic process of living and working on the island, gave us a rich understanding of the community and the way it experiences energy supply, and also painted a picture of the types of technologies that may be suitable for the community.

\section{B. Theme Elicitation Workshop}

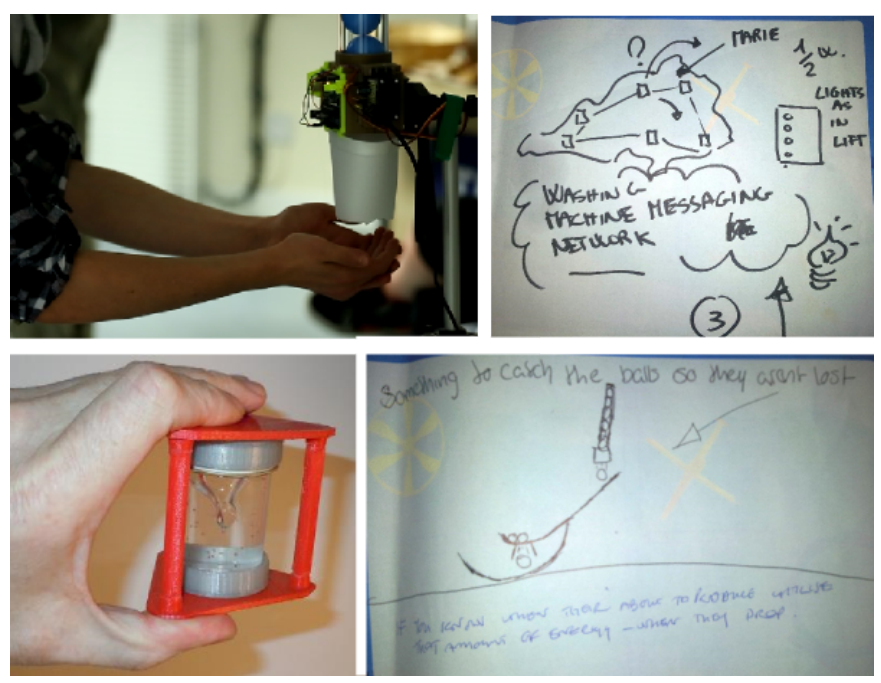

Fig. 2: Artefacts used during first workshop (left) and sample output produced (right).

1) Overview: The theme elicitation workshop was intended to engage with adults and teenagers on the island, encourage ideation around the utilisation of a variable energy supply, and help position the participants within the presented problem space.

In total, 16 participants attended the workshop, ranging in age from 16-60, and from different backgrounds including a local builder, artist, and a self-described digital crofter. As this was the first workshop to involve members of the community, the physical artefacts presented were intended to introduce the problem to the participants and start the elicitation of themes. The following describes the workshop tasks.

- Warm-up task. The main purpose of the warm-up task was to level technological understanding between the participants and the facilitators. Borrowing from the NeutralZone Metaphor technique [27], Inter-dimensional Energy was presented to the participants as a fictional power source that suffered from intermittent black-outs-akin to the island's power infrastructure. As this technology was first introduced to participants here, all had an equal understanding of it. It was represented as a small glass vial enclosed in a 3D printed plastic manifold (see bottom-left Figure 2), and the participants were asked to imagine ways that they may harness it as an energy source.

- Scenario-based game. As supply-driven energy use is not the currently established model of energy consumption, a way was needed to present it in a manner that was both 
understandable and interesting to different backgrounds. In order to achieve this, the Ping Pong Power (PPP) game was developed to help the participants better visualise variable energy supply. The aim of the game involved the participants needing to power a number of household appliances using dispensed ping pong balls (energy) (see top-left Figure 2). To gradually ease participants into the problem context, rules were slowly introduced to the game to mimic the properties of variable supply.

- Theme generation task. The aim of the generative task was to have the participants reflect on the previously played PPP game. Furthermore, this task allowed the participants to jointly explore the problem space and elicit some high-level themes that the community was willing to engage with. As the PPP game was a metaphor for a variable energy supply, we asked the participants to imagine ways to better utilise the energy/balls being released by the ping pong ball dispensers. During this task, the participants were encouraged to write down any ideas they had.

2) Output: This workshop resulted in a number of sketches and comments representing ways in which the energy/balls could be better utilised within the allotted time. A number of common themes emerged around the mechanisms and processes related to the storage, forecasting, and prediction of energy. A sample of these themes are shown in Figure 2.

\section{Design Constraint Workshop}

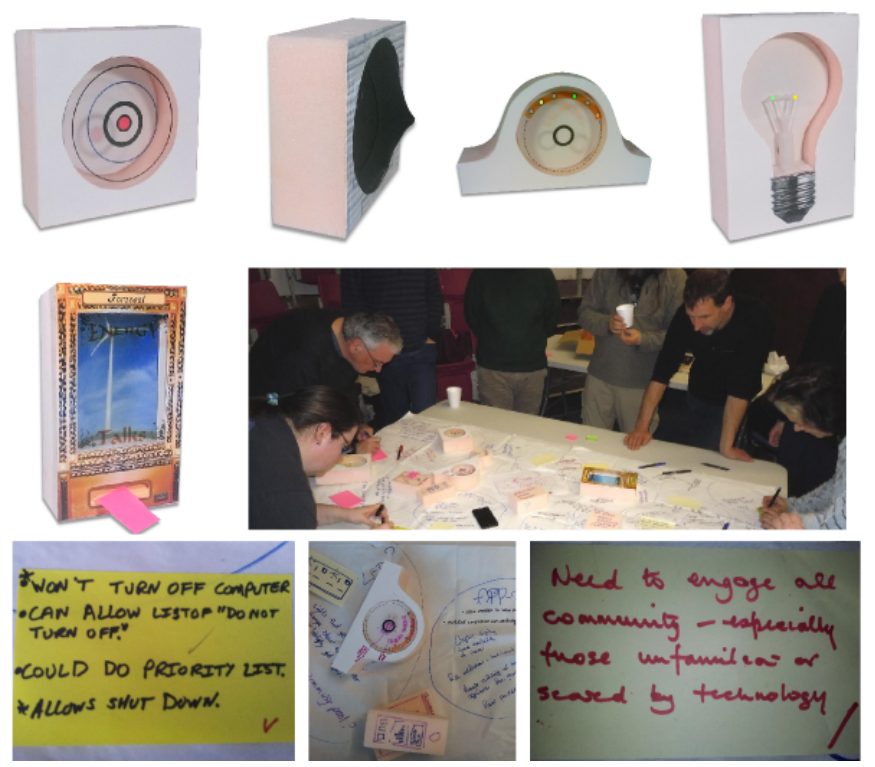

Fig. 3: Artefacts used during second workshop (top and middle-left) and sample output produced (bottom).

1) Overview: In total, 8 participants were involved, the majority of which were invited from the previous workshop based on their continued correspondence with us, whilst a couple of additional participants joined us from our other engagements and interviews.
Building upon the themes generated in the first workshop, a number of low-fidelity prototypes sketches were produced for the workshop; these are shown in Figure 3, and resembled a range of objects from clocks to fortune-telling machines found at fairgrounds.

The sketches were made from a combination of low fidelity materials, such as paper, printable annotate, and modelling foam as a way to decrease their perceived value; this was done so that the participants would feel free to draw on, alter, and potentially discard them.

Once we had briefly described the artefacts and the themes that inspired them, the participants were encouraged to write their thoughts on the table, which was covered in a paper tablecloth; an in-depth description of the artefacts can be found in our previous work [28].

Further evidence of the artefacts being used to encourage ideation can be seen in comments written on the table worksheet, such as "clock = great, [needs] forecasting in realtime" and "It should have tactile feedback for the sight/hearing impaired".

2) Output: From the initial themes brought forward from the first workshop, over 60 individual design artefacts were generated by the participants, including sketches and comments drawn on the main worksheet. Many of these artefacts took the form of early requirements, bringing us closer to the design of a digital mechanism to support the shifting of energy consumption; in total, 16 high-level requirements were generated. In addition, several form factors in which these requirements could manifest were suggested by the participants-these ideas ranged from mobile devices (i.e. phones and tablets), to more abstract, ambient devices.

These requirements gave us a clearer understanding of the main concerns held by the community, namely, fear of control being taken from the user in operating appliances-akin to the energy tariff offered by Scottish Hydro called Total Heating Total Control (THTC), which uses remote controlled electric storage heaters to synchronise use with available off-peak electricity [29]. Although the participants were interested in greater (potentially remote) control over their appliances (" [...] make things easier to turn off "), they were also concerned that same control could be used by external actors.

\section{Design Refinement Workshop}

1) Overview: In addressing the emerging requirements, we developed a prototype for shared public spaces (e.g. the school, the community centre) that allowed us to explore ambient displays of live and forecast energy production, called the Datarium. The Datarium resembles a house plant (see Figure 4) whose fronds twist and light up depending on the energy production level (calculated from the weather forecast). Datarium shows a live estimate of the electricity being produced by Tilley, and a forecast of the next days' production if interacted with.

The purpose of the refinement workshop session was to inspire the participants with the Datarium, and provide critical 


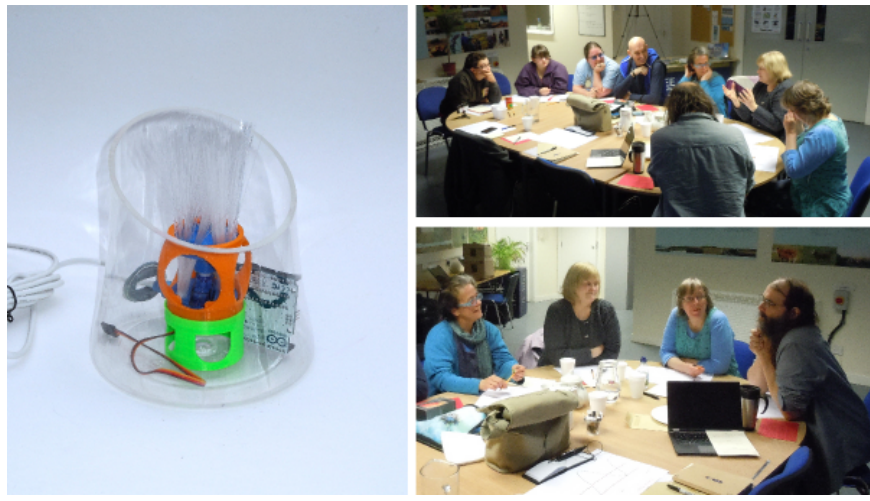

Fig. 4: The Datarium (left) and discussion between participants.

feedback and discussion, whilst drawing in on the constraints defined in the previous session.

Although the overall reception of the Datarium was in the main positive, it was clear that the participants were in favour of a more pragmatic and less ambiguous approach to communicating the availability of renewable energy. In fact, after viewing the ambient device, several participants noted that they would rather have the mechanism integrated with an existing piece of technology, rather than have an additional device in the house:

"For me, it's not the kind of thing I would want. I think something that'd flash on the television screen or your computer screen [...] as that's where people spend most of their visual time"-Participant $M$

Participant $M$ then elaborated on the possible use of mobile phones, as children also have access to mobile phones. Although an equal number of participants liked the idea of a stand-alone device, there was a consensus within the group for a smaller, portable device.

2) Output: From this workshop, we clarified some of the requirements elicited in the previous workshop-in this case, the community were curious about Datarium, but were resistant to the idea of an ambient, interpretive display, and were more receptive towards a pragmatic visualisation of data (i.e. charts). Building on this and requirements elicited in the previous workshops we started the development of another prototype: the Tiree Energy Pulse.

\section{Tiree Energy Pulse}

\section{A. Overview}

Based on the feedback about the Datarium, it was clear that the community were interested in a more easily interpreted mechanism for presenting the availability of renewable energy. As one of Datarium's detractors noted somewhat directly during the final workshop, Datarium was:

"It's missing two things for me... it's missing a pur-
pose..., and it's missing an incentive..."-Participant
$R$

There was also a desire for this mechanism to be smaller, more portable, and as Participant $M$ suggested, something that integrated with an existing appliance (such as the television or computer). One suggestion was an app for a mobile phone, which was felt to appeal to the younger generation.

The participants were also interested in more detailed forecasting of available renewable energy with availability presented with specific times and days.

This led to the Tiree Energy Pulse (TEP) web application. TEP emerged as a result of our contextual knowledge of the island combined with requirements and constraints elicited during the workshops, and critiques from domain relevant literature, such as [30]. Rather than eco-feedback, TEP is designed to layer on top of existing engagement around the weather and weather forecasting, ideally supporting the planning of energy intensive everyday activities around the availability of renewable energy.

TEP is a renewable energy forecasting web application that uses local weather data obtained from the Met office $\mathrm{API}^{2}$ to estimate the energy produced by Tilley for the next five days. Wind speed is compared to a look-up table of $\mathrm{kW}$ values supplied by the E-44 Enercon wind turbine datasheet ${ }^{3}$, which is then plotted onto a number of charts and bar visualisations (see Figure 5).

The development of the TEP protoype, along with reflections on the implications for design of eco-feedback and ecoforcasting HCI systems is detailed by Simm et al [31]. A summary of the prototype and the emergence of features from a Social SE perspective is included here as it emerged from the DivingBoard design thinking component of Speedplay.
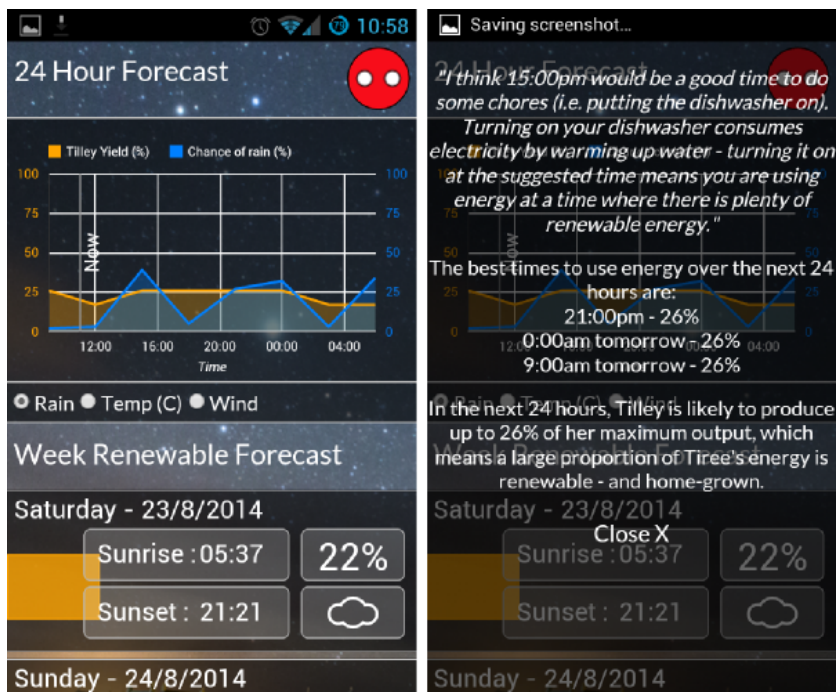

Fig. 5: Screenshots of the final version of Tiree Energy Pulse.

With TEP, we wanted to build on the existing unique relationships between the island community and the local weather, and provide a clearer, less ambiguous form of information display. A functional (rather than financial) incentive was used

${ }^{2}$ Met office API—http://www.metoffice.gov.uk/datapoint, accessed on April 7, 2015.

${ }^{3}$ Turbine specification-http://www.enercon.de/en-en/60.htm, accessed on April 7, 2015. 
in overlaying a weather forecast with the energy forecast. Islanders are frequently dependent on the weather, local weather information (e.g. chance of precipitation, temperature, wind speed etc.) was displayed against the energy generated.

In addition to the chart visualisations, the application also offered a digital assistant functionality that gave written forecast and household chore suggestions; this feature was added during the later iterations of the development cycle (see Section VI).

\section{B. Development and Deployment}

TEP was iteratively developed with a group of 8 households over a period of 5 weeks. There were continuous cycles of development and feedback, the first of which was conducted as an interview during deployment. Each iteration would conclude with an interview with each participant (one for each household), where they were asked a number of questions regarding the latest changes made to TEP and their energy usage habits. Part of each interview involved bringing into the conversation suggestions and comments made by other participants, which allowed us to receive multiple feedback points about potential new features and changes. At the close of the study, a discussion group was held on Tiree with participants from four households. This process was particularly useful for system feature prioritasation; one of the participant, for example, reported an interest in overlaying forecast energy data with live energy mix information from the grid. Although two other participants also expressed some interest in this aspect, this feature was given a low priority by the majority as it was felt that it would detract from the main purpose of the application. Table I describes the main changes made during each version of TEP ('implicit' is used where the feature was not explicitly asked for, but derived from observations and references made during conversation).

After the first iteration, the majority of new features reflected the feedback given by the lead users during the user trial. Towards the end of the second iteration, a new feature was added in the form of a digital assistant providing informal suggestions to energy usage in text format. It was hoped that presenting forecast data as text would improve the accessibility of the application to those not used to charts. The assistant was given the moniker Blinky in response to feedback previously captured about the Datarium, which was often likened to a small creature to be nurtured through mindful changes in energy consumption patterns.

"It is quite useful to have that extra way of digesting the information"-Participant $F$

Although we were able to meet with the participants at the beginning and end of the deployment, we weren't able to be present on the island during the development of TEP. This presented a number of challenges for communication, mainly due to the poor quality, low-speed, and sometimes absent broadband present on the island. Despite this, we were able to conduct weekly telephone interviews and ask the participants about their usage of TEP and feedback regarding current and new features.

\section{REFLECTION}

\section{A. Developing a Community Relationship}

One of the results of exploring a problem space with a community group is the cultivation of a relationship where all participants can feel free to contribute to the design and development process. An example of the developing relationship can be seen in the active communication observed on the part of the lead users. Although we didn't intentionally encourage the participants to contact us should they find a problem with the application, we found that we received several emails with informal error reports (see Table I).

\section{"Still loving the app! I tried to tap on the little Blinky today and the [phone brand] started logging an error for each tap. Seems Blinky is on the fritz!"- Participant $J$}

These reports would lead to a small fix being released before the end of a cycle, and would sometimes result in the early release of an already finished feature - this can be seen during iteration 2 of TEP development.

Through the relationship built with the island community, we were also able to encourage interest in the prototypes developed through a psychological ownership of them. Psychological ownership is the level of personal investment in the artefacts themselves [32], which can be used to help sustain an interest in the project beyond its completion. We found that interest in TEP was cultivated through the involvement of community members in its development.

"I think it's jolly, I think it's nice to see it evolving as well. It's been interesting, seeing things being added."-Participant $F$

We also found that some of the participants noted a change in their behaviour-both intended and unintended. In the case of the former, one of the participants reported that they would often use the TEP application to plan their chores according to the predicted weather.

"I've been planning my laundry by just turning on the [phone brand], you know instead of going to the $B B C$ website and 'faffing' about"-Participant J

Although this quote suggests that the user is already interested in synchronizing their energy use to that generated by renewable sources, it also demonstrates that TEP provides access to the information used to do so, and in a way more convenient than their prior sources. However, it is also important to note that although TEP was very well received by the participants, a few of our lead users reported that they had neglected to check the application as often as they would have liked to. When one participant was asked whether they still used the application, they commented-

\section{"I am, but I am surprised at how little I'm using it"-Participant $R$}

This lack of habitual use could be attributed to the novelty effect, although the participant suggested that they used TEP on the physical deployment more than the phone in their pocket because it was always physically visible. Despite 
TABLE I: CHANGE LOG OF THE TEP APPLICATION THROUghout ITS DEVELOPMENT. EACH NEW FEATURE IS PRESENTED ALONGSIDE THE INSPIRING DESIGN ARTEFACT OR REQUIREMENT.

\begin{tabular}{|c|c|c|c|}
\hline Iteration & Version & Change & Requirement/Prompt \\
\hline 1 & 0.01 & $\begin{array}{l}\text { 1) Line chart with Tilley output estimate for the next day. } \\
\text { 2) Weather overlay switchable between rain/wind/temp. } \\
\text { 3) } 5 \text { day bar chart for Tilley output. } \\
\text { 4) Data derived from MET office API. }\end{array}$ & $\begin{array}{l}\text { 1) It should provide a graphical display of available power. } \\
\text { 2) (Implicit) Common topic of conversation with Tiree Community. } \\
\text { 3) (Implicit) Themes of forecasting recurrent throughout workshops. } \\
\text { 4) (It should) display updates in real-time. }\end{array}$ \\
\hline 2 & 0.02 & $\begin{array}{l}\text { 1) Added weather overlay to following } 4 \text { days of forecast } \\
\text { 2) Added 'Now' line to chart to indicate current time } \\
\text { 3) (Bug Fix) Added message when Met API is unreachable. }\end{array}$ & $\begin{array}{l}\text { 1) (Implicit) (Week forecast) [...] would like overlay of weather on this. } \\
\text { 2) (Email) Perhaps a simple vertical line to show where we are time wise? } \\
\text { 3) (Email) Themes of forecast recurrent throughout workshops. }\end{array}$ \\
\hline 2 & 0.03 & $\begin{array}{l}\text { 1) Added "Blinky"-a digital helper that tells you when the } \\
\text { best point to use energy during the day, and suggests a } \\
\text { chore (e.g. use washing machine). }\end{array}$ & $\begin{array}{l}\text { 1) (Implicit) Desire indicated for tamogocthi-like feature (emotional at- } \\
\text { tachment) and a task scheduler-"whens the best time to do washing?. } \\
\text { It [should] engage all [of the] community. }\end{array}$ \\
\hline 3 & 0.03 .1 & $\begin{array}{l}\text { 1) Added server caching to the data obtained from MET (only } \\
5000 \text { calls can be made to the MET API per day). } \\
\text { 2) (Bug Fix) Fixed estimates given by Blinky. }\end{array}$ & $\begin{array}{l}\text { 1) (Design Constraint) Limit of } 5000 \text { calls to Met DataPoint API can be } \\
\text { made per day on current account. } \\
\text { 2) (Email) [... Seems Blinky is on the fritz! }\end{array}$ \\
\hline 3 & 0.03 .2 & $\begin{array}{l}\text { 1) (Bug Fix) Updated Blinky to give better (more useful) } \\
\text { suggestions for chores (no more washing at } 3 \mathrm{am} \text { ). } \\
\text { 2) The selected radio button (weather) selection is retained } \\
\text { within client cookie. }\end{array}$ & $\begin{array}{l}\text { 1) (Email) [...] Also, time of day? Telling me that } 3 A M \text { is the best time to } \\
\text { do laundry makes me giggle at it. } \\
\text { 2) [...] would like to be able to set "wind/rain/temp" and have it persist } \\
\text { between sessions. }\end{array}$ \\
\hline 4 & 0.04 & $\begin{array}{l}\text { 1) Added ability to see breakdown of day when the bar is } \\
\text { clicked by the user. } \\
\text { 2) Added a list of 'good times' to use energy via Blinky } \\
\text { dashboard. }\end{array}$ & $\begin{array}{l}\text { 1) [...] would like more interaction with elements (clicking on bars to see } \\
\text { fine-grained forecast etc.) } \\
\text { 2) I would prefer a list, because if it gives } 1 \text { time [...] you are no better } \\
\text { off than you were before really. }\end{array}$ \\
\hline
\end{tabular}

mitigating the lack of physical visibility, the participant also noted that they were less likely to check it when the phone had gone into standby.

\section{"Yeah, but again I'm not using it a lot because it's a blank screen"-Participant $R$}

This type of feedback demonstrates a high level of honesty from the participants regarding the application, and was also seen in the previous workshop where Participant M jokingly stated that they would have "thrown it out of the window" if she was given the Datarium.

With regards to the physical artefacts produced through DivingBoard, there was evidence to suggest that they helped inspire ideation and acted as talking points between the participants. In addition, we found that the physical artefacts were effective at conveying an abstract idea or concept (such as energy availability). This was observed particularly in the first workshop, where despite concise descriptions of the project, the goals of the project only really became clear after having the participants learn through the physical workshop activities.

Finally, there was certainly a sense that the community appreciated that they were being involved in the exploration of the problem space and the design of the digital mechanisms to address it. This sentiment is best captured by one of the participants during the second workshop:

"[...] I think for me, this whole thing shows the importance of how productive collaboration can be, and how it can facilitate this kind of brainstorming session [...]"-Participant $B$

User participation has been key to the development of a digital intervention that the community feels appropriate to the context on Tiree. From the outset of the first workshop, a number of possible ideas emerged that the participants were interested in exploring. In the workshops that followed, these ideas were explored, evolved and would sometimes lead to dead-ends-ideas that although still valid, were not explored further in the project. One idea that evolved through prototypes was energy forecasting, first appearing in the Datarium and evolving through the TEP smartphone app, however development of the physical form ended.

In addition, through the ethnographic approach taken to understand the context the system would operate in, researchers built a deep understanding of the community they were researching with. This not only lead to the selection of the type of mechanism that the islanders were receptive to, but also the elicitation of requirements that could be considered appropriate for both the domain and the community.

\section{B. Lessons Learnt}

From working with the Tiree community, we have learnt a number of valuable lessons that have come from applying design thinking through DivingBoard to a community-driven project; these lessons are:

- Embrace open-ended enquiry. Whilst formal methods can provide a recipe for the successful development of an ICT system where requirements are well defined, we believe that Social Software Engineering mandates a less disciplined, more exploratory approach where the context of the system needs to be explored. Our experience with the Tiree community shows that such an informal process can result in the elicitation of appropriate requirements to tackle tough societal problems. Indeed, our approach fostered a culture where participants felt they were involved in the design process and were able to contribute.

- Qualitative data adds depth. While user stories are intended to capture all aspects of a requirement, they can miss out crucial non-functional properties [33]. In DivingBoard, requirements elicited from the workshops are supplemented by in depth interviews with each of the participants, both prior to and during the development cycle, to ground these requirements in the context of 
the island. This was reflected by the emphasis placed on weather in TEP-weather and the resilience of island life in response to it, was a recurring powerful theme despite not be explicitly stated as requirements.

- Trust plays an important role. In the context of Social $\mathrm{SE}$, there are several trust-based challenges to overcome when developing systems with community groups in a participatory sense-this is particularly apparent in small communities, where they can be exclusive, even distrust$f u l$, of outsiders and their intentions [34]. Indeed, this potential lack of trust may result in the community "not articulating felt needs, and instead giving answers that the incomer wants to hear" [35]. Through the relationship we built with the island community and the time invested in getting to understand the community, we found that the participants were forthcoming with feedback, and they felt that they were able provide critical input.

\section{RELATED WORK}

There has been a movement within the SE community to draw upon the natural creativity of the end user rather than just the system engineers. Much of this work focuses on using creative workshops, such as that of Maiden et al. [5], [36], which present the use of creativity workshops to elicit requirements for various air traffic control systems. Requirements elicitation through creative workshops is further demonstrated by Schlosser et al., who describe the development of an event database application [37]. Whilst such approaches show the value of creativity in a participatory space, these approaches often select participants with domain expertise, and do not investigate whether such approaches can be applied to groups with little or no experience in the problem domain.

Scenarios also play a part in inspiring creativity within a creative workshop setting, ranging from storyboarding to immersing the participants within a fictionalised world. The former has been used by the SE community for some time [25], although there is ongoing work investigating how they can be used as a tool to encourage the elicitation of requirements in a fun and engaging way. Williams and Alspaugh [38] suggest that incorporating comic book styling into the creation of storyboards may help participants express their desires earlier in the requirements process. Whilst this may be useful in helping requirements boil to the surface, the work suggests that it is intended to encourage the elicitation of requirements that may be already known but not expressed, rather than to inspire new requirements entirely.

At the other end of the spectrum, the approach by McGinley and Nakata [39] uses physical artefacts as part of creative workshops to form a science fiction scenario, which is used as a way to engage the participants. Indeed, physical artefacts play an important role in the process of design, as they provide support for creativity and ideation to the participants and practitioners alike [40]. However, whilst many approaches present artefacts as focal points for participants to use in the design process, none of these approaches develop and iteratively build upon these artefacts based on feedback from the participating actors.

Briefly, we contextualise our work in terms of energy sustainability. Touching upon the themes of scenario-driven methods, Rodden et al. [41] investigated user perception of software agents operating within a future smart grid by using sketches to illustrate such a world. Whilst this approach is fundamentally different to the one we describe, their observations align well with our findings-i.e. a negative reaction was noted towards systems that take total control away from the user. Strengers [30] critiques eco-feedback systems as casting users as rational resource managers, and argues for broader consideration of the role of energy in everyday life. Drawing inspiration from this, TEP augments existing weather forecasting with energy availability to support planning, rather than simply providing instantaneous eco-feedback.

\section{CONCLUSION}

The problems unearthed by social SE are invariably complex and sometimes wicked; they present loosely formulated, persistent, and fluid problems that cannot be addressed through formal methods and analysis alone. As these problems often have potentially critical connotations, user participation is key to ensure that those on the ground play a part in the development of any solution. This poses several challenges: How do you explore a complex problem with a community group that has little or no experience in the target domain? and 2) once the problem domain is jointly understood, how do you begin to elicit requirements and envisage technological solutions together?

Our contribution to Social SE is embedding design thinking in an agile and participatory framework [1] that is applied to a poorly-understood complex socio-technical problems. We argue that by doing so this approach provides a powerful mechanism for the generation and development of software solutions to underspecified and open ended problems. Congruous with this, we also effectively use physical artefacts in developing a shared understanding with our stakeholders. Specifically this paper has contributed DivingBoard, a design thinking component of the SE process that embraces a potentially openended exploration of the problem domain. Through OnSupply we described how DivingBoard generates well-contextualised domain specific artefacts and ideas in an iterative manner in the spirit of the agile manifesto.

Numerous prototypes were iteratively developed with the Tiree community, including the Datarium and TEP which jointly explore the presentation of live and future renewable energy availability. The results of this work demonstrate that creativity-driven workshops and physical artefacts are effective tools in encouraging the generation of requirements and solutions for complex problems.

We would note that wicked social software engineering problems do not have a single or indeed purely technical solution, hence we need a process that allows us to explore multiple ways of addressing different manifestations of the same problem. Our approach is to integrate design thinking 
into a Social SE framework. The implication for SE is that such problems need to be approached by being mindful of "technological solutionism" which tends to treat complex problems in often a reductive and simplistic way.

\section{ACKNOWLEDGEMENTS}

We are grateful to the islanders of Tiree and Tiree Development Trust for their participation. We gratefully acknowledge our funders EPSRC via Citizens Transforming Society: Tools for Change (Catalyst project) ref. EP/I033017/1.

\section{REFERENCES}

[1] M. A. Ferrario, W. Simm, P. Newman, S. Forshaw, and J. Whittle, "Software Engineering for 'Social Good': Integrating Action Research, Participatory Design, and Agile Development," in ICSE. NY, USA: ACM, 2014, pp. 520-523.

[2] S. M. Easterbrook, "From Computational Thinking to Systems Thinking: A conceptual toolkit for sustainability computing," in Proc. ICT4S, 2014.

[3] W. Simm, M. A. Ferrario, A. Gradinar, and J. Whittle, "Prototyping'clasp': implications for designing digital technology for and with adults with autism," in Proc. DIS. ACM, 2014, pp. 345-354.

[4] J. Southern, R. Ellis, M. A. Ferrario, R. McNally, R. Dillon, W. Simm, and J. Whittle, "Imaginative labour and relationships of care: $\mathrm{Co}-$ designing prototypes with vulnerable communities," Technological Forecasting and Social Change, vol. 84, pp. 131-142, 2014.

[5] N. Maiden, C. Ncube, and S. Robertson, "Can requirements be creative? experiences with an enhanced air space management system," in ICSE, May 2007, pp. 632-641.

[6] H. Rittel and M. Webber, "Dilemmas in a General Theory of Planning," Policy Sciences, vol. 4, no. 2, pp. 155-169, 1973.

[7] M. M. Burnett and B. A. Myers, "Future of End-user Software Engineering: Beyond the Silos," in FOSE. NY, USA: ACM, 2014, pp. 201-211.

[8] P. Newman, S. Forshaw, W. Simm, M. Ferrario, J. Whittle, and A. Friday, "Seeding the Design Process for Future Problems," in Human-Centered Software Engineering, 5th International Conference. Springer, 2014.

[9] S. Wilson, M. Bekker, H. Johnson, and P. Johnson, "Costs and Benefits of User Involvement in Design: Practitioners Views," in People and Computers XI, M. Sasse, R. Cunningham, and R. Winder, Eds. Springer London, 1996, pp. 221-240.

[10] K. Michlewski, "Uncovering design attitude: Inside the culture of designers," Organization Studies, vol. 29, no. 3, pp. 373-392, 2008.

[11] U. Abelein, H. Sharp, and B. Paech, "Does involving users in software development really influence system success?" Software, IEEE, vol. 30, no. 6, pp. 17-23, 2013.

[12] K. Beck, M. Beedle, A. van Bennekum, A. Cockburn, W. Cunningham, M. Fowler, J. Grenning, J. Highsmith, A. Hunt, R. Jeffries, J. Kern, B. Marick, R. C. Martin, S. Mellor, K. Schwaber, J. Sutherland, and D. Thomas, "Manifesto for Agile Software Development," 2001.

[13] E. Brandt, "Designing Exploratory Design Games: A Framework for Participation in Participatory Design?" in PDC. NY, USA: ACM, 2006, pp. 57-66.

[14] S. Chamberlain, H. Sharp, and N. Maiden, "Towards a Framework for Integrating Agile Development and User-centred Design," in $X P$ ' 06 . Berlin, Heidelberg: Springer-Verlag, 2006, pp. 143-153.

[15] K. Kautz, "Participatory Design Activities and Agile Software Development," in Human Benefit through the Diffusion of Information Systems Design Science Research. Springer Berlin Heidelberg, 2010, vol. 318, pp. 303-316.

[16] C. Hansson, Y. Dittrich, and D. Randall, "How to Include Users in the Development of Off-the-Shelf Software: A Case for Complementing Participatory Design with Agile Development," in System Sciences, 2006. HICSS '06. Proceedings of the 39th Annual Hawaii International Conference on, vol. 8, Jan 2006, pp. 175c-175c.

[17] C. Spinuzzi, "The Methodology of Participatory Design," Technical Communication, vol. 52, no. 2, pp. 163-174, 2005.

[18] E. B.-N. Sanders and P. J. Stappers, "Co-creation and the New Landscapes of Design," Co-design, vol. 4, no. 1, pp. 5-18, 2008.
[19] D. Pagano and B. Brügge, "User involvement in software evolution practice: A case study," in ICSE. Piscataway, NJ, USA: IEEE Press, 2013, pp. 953-962.

[20] R. Subramanyam, F. L. Weisstein, and M. S. Krishnan, "User participation in software development projects," Commun. ACM, vol. 53, no. 3, pp. 137-141, Mar. 2010.

[21] R. Buchanan, "Wicked Problems in Design Thinking," Design Issues, vol. 8, no. 2, pp. 5-21, 1992.

[22] R. Coyne, "Wicked Problems Revisited," Design Studies, vol. 26, no. 1, pp. $5-17,2005$.

[23] G. R. Hayes, "The Relationship of Action Research to Human-computer Interaction," ACM Trans. Comput.-Hum. Interact., vol. 18, no. 3, pp. 15:1-15:20, Aug. 2011.

[24] S. Christopher, V. Watts, A. K. H. G. McCormick, and S. Young, "Building and Maintaining Trust in a Community-based Participatory Research Partnership," American Journal of Public Health, vol. 98, no. 8 , p. $1398,2008$.

[25] H. Sharp, H. Robinson, and M. Petre, "The Role of Physical Artefacts in Agile Software Development: Two Complementary Perspectives." Interacting with Computers, vol. 21, no. 12, pp. 108 - 116, 2009, special issue: Enactive Interfaces Physicality \& Interaction.

[26] D. Kolb, Experiential learning: experience as the source of learning and development. Prentice-Hall, 1984

[27] S. Forshaw, L. Cruickshank, and A. Dix, "Collaborative Communication Tools for Designing: Physical-Cyber Environments," in Proc. of 26th BCS Conference on Human Computer Interaction, 2012.

[28] M. Ferrario, S. Forshaw, P. Newman, W. Simm, A. Friday, and A. Dix, "On the Edge of Supply: Designing Renewable Energy Supply into Everyday Life," in Proc. ICT4S. Atlantis Press, July 2014.

[29] O. Edberg and C. Naish, "Energy Storage and Management Study," AEA Technology PLC, Tech. Rep., October 2010.

[30] Y. A. Strengers, "Designing Eco-feedback Systems for Everyday Life," in Proc. CHI. NY, USA: ACM, 2011, pp. 2135-2144.

[31] W. Simm, M. A. Ferrario, P. Newman, A. Friday, S. Forshaw, A. Dix, and M. Hazas, "Tiree Energy Pulse: Exploring Renewable Energy Forecasts on the Edge of the Grid," in Proc. CHI. NY, USA: ACM, 2015.

[32] J. L. Pierce, T. Kostova, and K. T. Dirks, "The State of Psychological Ownership: Integrating and Extending a Century of Research," Review of general psychology, vol. 7, no. 1, p. 84, 2003.

[33] L. Buglione and A. Abran, "Improving the User Story Agile Technique Using the INVEST Criteria," in Software Measurement and the 2013 Eighth International Conference on Software Process and Product Measurement (IWSM-MENSURA), 2013 Joint Conference of the 23rd International Workshop on, Oct 2013, pp. 49-53.

[34] J. Garland and N. Chakraborti, "Race, Space and Place: Examining Identity and Cultures of Exclusion in Rural England," Ethnicities, vol. 6, no. 2, pp. 159-177, 2006.

[35] A. Dearden and S. M. H. Rizvi, "Adapting Participatory and Agile Software Methods to Participatory Rural Development." in Proc. PDC, D. Hakken, J. Simonsen, and T. Roberston, Eds. ACM, 2008, pp. 221-225.

[36] N. Maiden, S. Manning, S. Robertson, and J. Greenwood, "Integrating Creativity Workshops into Structured Requirements Processes," in Proc. DIS. NY, USA: ACM, 2004, pp. 113-122.

[37] C. Schlosser, S. Jones, and N. Maiden, "Using a creativity workshop to generate requirements for an event database application," in Requirements Engineering: Foundation for Software Quality, ser. Lecture Notes in Computer Science, B. Paech and C. Rolland, Eds. Springer Berlin Heidelberg, 2008, vol. 5025, pp. 109-122.

[38] A. Williams and T. Alspaugh, "Articulating software requirements comic book style," in Multimedia and Enjoyable Requirements Engineering Beyond Mere Descriptions and with More Fun and Games, 2008. MERE '08. Third International Workshop on, Sept 2008, pp. 4-8.

[39] T. McGinley and K. Nakata, "A Participatory Design Approach to the Wicked Problem of Designing Sustainable Communities," The TSBE EngD Conference, 2012.

[40] D. Vyas, D. Heylen, A. Nijholt, and G. van der Veer, "Experiential Role of Artefacts in Cooperative Design," in Proc. of the Fourth International Conference on Communities and Technologies. NY, USA: ACM, 2009, pp. 105-114.

[41] T. A. Rodden, J. E. Fischer, N. Pantidi, K. Bachour, and S. Moran, "At Home with Agents: Exploring Attitudes Towards Future Smart Energy Infrastructures," in Proc. CHI. NY, USA: ACM, 2013, pp. 1173-1182. 